\title{
Vector diffraction theory of refraction of light by a spherical surface
}

\author{
Shekhar Guha and Glen D. Gillen* \\ Materials and Manufacturing Directorate, Air Force Research Laboratory, Wright-Patterson Air Force Base, \\ Dayton, Ohio 45433, USA
}

Received March 17, 2006; accepted August 11, 2006;

posted September 1, 2006 (Doc. ID 69022); published December 20, 2006

\begin{abstract}
Focusing of light by a curved surface is described using the vector Kirchhoff diffraction theory. The electromagnetic fields of a light beam incident as a plane wave on a curved surface separating two transparent media having different refractive indices are expressed as dimensionless double integrals. The integrals are evaluated for a few specific cases, and the three-dimensional distribution of irradiance near the focus is determined. The role of aberration in limiting the maximum achievable irradiance is studied. The distribution of the longitudinal components of the electric field in the focal region is also studied, and the region where the longitudinal fields maximize is determined. (c) 2006 Optical Society of America OCIS codes: $050.1960,080.1010$.
\end{abstract}

\section{INTRODUCTION}

Rayleigh-Sommerfeld and scalar Kirchhoff beam propagation formalisms are convenient ways to describe the propagation of light from a plane surface (where the light fields are known) to a point of interest beyond that surface. These methods can easily be adapted to include curved optical elements placed along the axis of propagation if the optical elements are thin and paraxial conditions apply. ${ }^{1}$ However, these beam propagation models cannot be used for high-numerical aperture lenses or curved mirrors that are commercially available and used in a wide variety of optical applications. Additionally, the scalar nature of these beam propagation models do not account for the interdependence of the different components of the electromagnetic fields. To accurately model the propagation of light where the usual thin lens and paraxial approximations do not apply, one needs a more rigorous approach that incorporates the full-vector nature of electromagnetic radiation.

Vector components of the electromagnetic fields of light in the focal region had been determined previously by $\mathrm{Ri}$ chards and Wolf $^{2}$ for the case of an aplanatic, i.e., aberration-free, converging beam. Visser and Wiersma ${ }^{3}$ and $\mathrm{Hsu}$ and Barakat ${ }^{4}$ have considered the case of aberrated incident phase fronts but not the case of refraction between two media. We use here the vector diffraction theory of Stratton and $\mathrm{Chu},{ }^{5,6}$ which applies the vector analog of Green's theorem to determine the electromagnetic fields at a point inside a closed volume given the fields on the curved surface.

The case of a linearly polarized plane wave of light traveling in one transparent medium and incident on a spherical surface separating the medium from a second one having a different refractive index is considered here. The values of the fields incident on the surface are known after a plane at which the incident wave has zero phase is defined. For highly curved surfaces, the angular dependence of the Fresnel transmission coefficients must be taken into consideration as the angle of incidence along the surface can also become large. With the Fresnel transmission factors and angular-dependent refraction effects, the values of the fields just inside the second medium are determined, and the vector diffraction integrals are then used to find the values of the fields at any point in the second medium in terms of integrals involving the values of the fields at the surface. The onset of spherical aberration is investigated along with the effects of spherical aberration on the maximum obtainable intensity. There are four main differences of this work from previous treatments of vector diffraction theory for focused light: (1) the theory and results are expressed in dimensionless units so as to be applicable to a wide range of laser and optical parameters, (2) the paraxial-type approximations used in Eq. (15) of Hsu and Barakat ${ }^{4}$ are not invoked, (3) detailed distributions of the longitudinal field components are determined for both the aberrated and the unaberrated cases, and (4) the relative contributions of the three different terms of the Stratton-Chu diffraction integral are investigated. Although the case considered here is that of a spherical surface and a plane incident wave, extension to cases of other curvatures and to incident beams having different spatial distribution, such as one with a Gaussian amplitude profile, would be straightforward.

\section{VECTOR DIFFRACTION THEORY AT A SPHERICAL SURFACE}

Suppose $n_{1}$ and $n_{2}$ denote the refractive indices of the two media and $\lambda$ denotes the (vacuum) wavelength of a plane wave of light traveling in the first medium and incident upon a convex spherical surface having a radius of curvature of $R_{1}$, as shown in Fig. 1 . We designate the polarization direction of the wave to be the $x$ direction and the direction of propagation to be the $z$ direction of a rectangular Cartesian coordinate system. For convenience, the spherical coordinates are also used here, with 


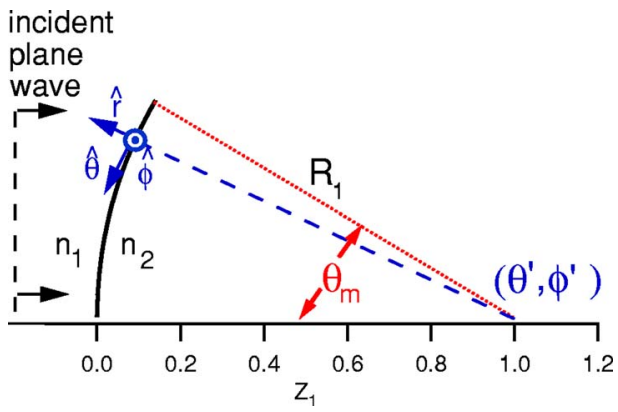

Fig. 1. (Color online) Illustration of the spherical boundary between media 1 and 2 ; the radius of curvature, $R_{1}$; the maximum angle of the surface, $\theta_{m}$; the polar angles to the surface source point, $\theta^{\prime}$ and $\phi^{\prime}$; and the unit vectors $\hat{r}, \hat{\theta}$ and $\hat{\phi}$.

the origin of the spherical coordinate system on the $z$ axis, located at the center of the spherical surface separating the two media, as shown in Fig. 1. The coordinates of the source points, i.e., the points on the surface, are denoted by primed variables, and those of the field points, i.e., the points in the second medium, are denoted by unprimed variables. The time-independent parts of the electric and magnetic fields incident upon the surface are given by

$$
\begin{aligned}
& \vec{E}\left(\vec{r}^{\prime}\right)=E_{0} \exp \left(-i k_{1} z^{\prime}\right) \hat{i}, \\
& \vec{H}\left(\vec{r}^{\prime}\right)=H_{0} \exp \left(-i k_{1} z^{\prime}\right) \hat{j},
\end{aligned}
$$

where $H_{0}=n_{1} E_{0} / Z_{0}$, with $Z_{0}=\sqrt{\mu_{0} / \epsilon_{0}}$, and $k_{1}=2 \pi n_{1} / \lambda$. Expressing the Cartesian unit vectors $\hat{i}, \hat{j}, \hat{k}$ in terms of the spherical coordinates $r^{\prime}, \theta^{\prime}, \phi^{\prime}$ and unit vectors $\hat{r}, \hat{\theta}, \hat{\phi}$ (also illustrated in Fig. 1), we can rewrite Eqs. (1) and (2) as

$$
\begin{aligned}
\vec{E}_{-}\left(\vec{r}^{\prime}\right)= & E_{0} \exp \left[-i k_{1} R_{1}\left(1+\cos \theta^{\prime}\right)\right] \\
& \times\left(\sin \theta^{\prime} \cos \phi^{\prime} \hat{r}+\cos \theta^{\prime} \cos \phi^{\prime} \hat{\theta}-\sin \phi^{\prime} \hat{\phi}\right), \\
\vec{H}_{-}\left(\vec{r}^{\prime}\right)= & H_{0} \exp \left[-i k_{1} R_{1}\left(1+\cos \theta^{\prime}\right)\right] \\
& \times\left(\sin \theta^{\prime} \sin \phi^{\prime} \hat{r}+\cos \theta^{\prime} \sin \phi^{\prime} \hat{\theta}+\cos \phi^{\prime} \hat{\phi}\right),
\end{aligned}
$$

where the minus subscripts of $\vec{E}$ and $\vec{H}$ represent the fields just outside the spherical surface, i.e., in the incident medium.

At every source point (say, denoted by $R_{1}, \theta^{\prime}$, and $\phi^{\prime}$ ), the spherical surface may be considered locally to be a plane surface, and the Fresnel transmission factors obtained for a plane wave incident upon a plane surface ${ }^{7}$ can be applied. Since the propagation direction of the incident plane wave is in the $z$ direction and the normal to the surface is along the radial $(\hat{r})$ direction, the plane of incidence is defined by the $\hat{r}$ and $\hat{k}$ vectors. By definition, the $\hat{\theta}$ vector lies in this plane, and the $\hat{\phi}$ vector is perpendicular to this plane. Therefore, in Eq. (3), the $\hat{r}$ and $\hat{\theta}$ components are parallel to the plane of incidence ( $p$ polarized), and the $\hat{\phi}$ component is perpendicular to the plane of incidence ( $s$ polarized).

Applying the appropriate Fresnel transmission coefficients from Ref. 7 and using the appropriate refraction ef- fects on the plane wave's $s$-polarization and $p$-polarization components, we can write the transmitted fields as

$$
\begin{aligned}
\vec{E}_{+}\left(\vec{r}^{\prime}\right)= & E_{0} \exp \left[-i k_{1} R_{1}\left(1+\cos \theta^{\prime}\right)\right] \\
& \times\left(T_{p} \sin \theta_{t}^{\prime} \cos \phi^{\prime} \hat{r}+T_{p} \cos \theta_{t}^{\prime} \cos \phi^{\prime} \hat{\theta}\right. \\
& \left.-T_{s} \sin \phi^{\prime} \hat{\phi}\right), \\
\vec{H}_{+}\left(\vec{r}^{\prime}\right)= & \frac{n_{2} E_{0}}{Z_{0}} \exp \left[-i k_{1} R_{1}\left(1+\cos \theta^{\prime}\right)\right] \\
& \times\left(T_{s} \sin \theta_{t}^{\prime} \sin \phi^{\prime} \hat{r}+T_{s} \cos \theta_{t}^{\prime} \sin \phi^{\prime} \hat{\theta}\right. \\
& \left.+T_{p} \cos \phi^{\prime} \hat{\phi}\right),
\end{aligned}
$$

where the plus subscripts denote the fields just inside the second medium. The Fresnel transmission coefficients in Eqs. (5) and (6) are

$$
\begin{aligned}
& T_{p}=\frac{2 n_{1} \cos \theta^{\prime}}{n_{1} \cos \theta^{\prime}+n_{2} \cos \theta_{t}^{\prime}}, \\
& T_{s}=\frac{2 n_{1} \cos \theta^{\prime}}{n_{2} \cos \theta^{\prime}+n_{1} \cos \theta_{t}^{\prime}},
\end{aligned}
$$

for the $p$ polarizations, respectively, and $\theta_{t}^{\prime}$ is the local angle of refraction into the transmitted medium, given by Snell's law; i.e.,

$$
\theta_{t}^{\prime}=\sin ^{-1}\left(\frac{n_{1}}{n_{2}} \sin \theta^{\prime}\right) .
$$

Care needs to be taken in evaluating the inverse sine in Eq. (9) to ensure that $\theta_{t}^{\prime}$ is obtuse if the incident angle $\theta^{\prime}$ is obtuse.

According to Huygen's principle, the fields in a bound, source-free region of space can be thought of as arising from spherical waves emanated by every point on the surface bounding that space. A generalization of this principle to the vector case is given in Eq. (22) in Stratton. ${ }^{6}$ For the choice of the time-independent component of the electromagnetic fields assumed in the treatment here, the vector Huygen's principle formulas in Rothwell and Cloud $^{8}$ are appropriate:

$$
\begin{aligned}
\vec{E}(\vec{r})= & -\int_{S} \mathrm{~d} a^{\prime}\left[-i \omega \mu\left(\hat{n}^{\prime}\right.\right. \\
& \left.\left.\times \vec{H}_{+}\right) G+\left(\hat{n}^{\prime} \times \vec{E}_{+}\right) \times \vec{\nabla}^{\prime} G+\left(\hat{n}^{\prime} \cdot \vec{E}_{+}\right) \vec{\nabla}^{\prime} G\right], \\
\vec{H}(\vec{r})= & -\int_{S} \mathrm{~d} a^{\prime}\left[i \omega \epsilon \left(\hat{n}^{\prime}\right.\right. \\
& \left.\left.\times \vec{E}_{+}\right) G+\left(\hat{n}^{\prime} \times \vec{H}_{+}\right) \times \vec{\nabla}^{\prime} G+\left(\hat{n}^{\prime} \cdot \vec{H}_{+}\right) \vec{\nabla}^{\prime} G\right],
\end{aligned}
$$

where the integrations extend over the surface $S$ enclosing the field point $\vec{r}$ and the values of $\vec{E}_{+}$and $\vec{H}_{+}$in the integrands are those evaluated just inside the second medium and given by Eqs. (5) and (6). The Green's function, $G$, used in Eqs. (10) and (11), is given by 


$$
G=\frac{\exp \left(-i k_{2} \rho\right)}{4 \pi \rho}
$$

where $k_{2}=2 \pi n_{2} / \lambda$ and

$$
\rho^{2}=\left|\vec{r}(x, y, z)-\vec{r}^{\prime}\left(x^{\prime}, y^{\prime}, z^{\prime}\right)\right|^{2}=\left(x-x^{\prime}\right)^{2}+\left(y-y^{\prime}\right)^{2}+\left(z-z^{\prime}\right)^{2},
$$

with $\vec{r}^{\prime}$ denoting the position vector of the source point and $\vec{r}$ denoting the position vector of the field point. The components of $\vec{r}$ and $\vec{r}^{\prime}$ can be expressed in terms of the spherical coordinates as

$$
\begin{gathered}
x=r \sin \theta \cos \phi, \\
y=r \sin \theta \sin \phi, \\
z=r \cos \theta, \\
x^{\prime}=R_{1} \sin \theta^{\prime} \cos \phi^{\prime}, \\
y^{\prime}=R_{1} \sin \theta^{\prime} \sin \phi^{\prime}, \\
z^{\prime}=R_{1} \cos \theta^{\prime},
\end{gathered}
$$

where $r$ is the distance of the field point from the origin.

\section{NORMALIZATION AND SIMPLIFICATION}

Dimensionless parameters, dimensionless functions, and normalized distances are used in this treatment for mathematical convenience and to allow the treatment to be applicable to a variety of chosen wavelengths and radii of curvature. The normalized distances are defined as

$$
\begin{gathered}
x_{1} \equiv \frac{x}{R_{1}}, \quad y_{1} \equiv \frac{y}{R_{1}}, \quad z_{1} \equiv \frac{z}{R_{1}}, \\
x_{1}^{\prime} \equiv \frac{x^{\prime}}{R_{1}}, \quad y_{1}^{\prime} \equiv \frac{y^{\prime}}{R_{1}}, \quad z_{1}^{\prime} \equiv \frac{z^{\prime}}{R_{1}}, \\
\rho_{1} \equiv \frac{\rho}{R_{1}} .
\end{gathered}
$$

Using a dimensionless parameter and a dimensionless function

$$
p_{2} \equiv \frac{2 \pi n_{2} R_{1}}{\lambda}, \quad G_{1} \equiv 4 \pi R_{1} G=\frac{\exp \left(-i p_{2} \rho_{1}\right)}{\rho_{1}}
$$

we obtain

$$
\vec{\nabla}^{\prime} G=\frac{i p_{2} G_{2}}{4 \pi R_{1}^{2}} \vec{r}_{11}
$$

where

$$
\vec{r}_{11} \equiv\left(x_{1}-x_{1}^{\prime}\right) \hat{i}+\left(y_{1}-y_{1}^{\prime}\right) \hat{j}+\left(z_{1}-z_{1}^{\prime}\right) \hat{k}
$$

$$
G_{2} \equiv-\frac{1}{i p_{2} \rho_{1}} \frac{\partial G_{1}}{\partial \rho_{1}}=\frac{G_{1}}{\rho_{1}}\left(1+\frac{1}{i p_{2} \rho_{1}}\right) .
$$

We also further define

$$
n \equiv \frac{n_{2}}{n_{1}}, \quad t_{1} \equiv \frac{T_{s}}{T_{p}}=\frac{\cos \theta^{\prime}+n \cos \theta_{t}^{\prime}}{\cos \theta_{t}^{\prime}+n \cos \theta^{\prime}}
$$

as the normalized refractive index and transmission coefficient and

$$
f_{2} \equiv \exp \left[-i k_{1} R_{1}\left(1+\cos \theta^{\prime}\right)\right]=\exp \left[-i p_{2}\left(1+\cos \theta^{\prime}\right) / n\right]
$$

as the phase of the fields just outside the spherical interface between the media. The fields in Eqs. (5) and (6) may be rewritten as

$$
\begin{aligned}
& E_{+}(\vec{r})=E_{0} f_{2} T_{p} \vec{e}_{1}(\vec{r}), \\
& H_{+}(\vec{r})=\frac{n_{2} E_{0}}{Z_{0}} f_{2} T_{p} \vec{h}_{1}(\vec{r}),
\end{aligned}
$$

where

$$
\vec{e}_{1}\left(\vec{r}^{\prime}\right) \equiv e_{r} \hat{r}+e_{\theta} \hat{\theta}+e_{\phi} \hat{\phi}
$$

$$
\vec{h}_{1}\left(\vec{r}^{\prime}\right) \equiv h_{r} \hat{r}+h_{\theta} \hat{\theta}+h_{\phi} \hat{\phi}
$$

with

$$
\begin{aligned}
& e_{r}=\sin \theta_{t}^{\prime} \cos \phi^{\prime}, \\
& e_{\theta}=\cos \theta_{t}^{\prime} \cos \phi^{\prime}, \\
& e_{\phi}=-t_{1} \sin \phi^{\prime}, \\
& h_{r}=t_{1} \sin \theta_{t}^{\prime} \sin \phi^{\prime}, \\
& h_{\theta}=t_{1} \cos \theta_{t}^{\prime} \sin \phi^{\prime}, \\
& h_{\phi}=\cos \phi^{\prime} .
\end{aligned}
$$

Substituting Eqs. (20), (25), and (26) into Eqs. (10) and (11) and using the relationships

$$
\begin{aligned}
i \omega \mu G \frac{n_{2} E_{0}}{Z_{0}}= & \frac{i p_{2} G_{1}}{4 \pi R_{1}^{2}} E_{0}, \\
i \omega \epsilon G E_{0} & =\frac{i p_{2} G_{1}}{4 \pi R_{1}^{2}} \frac{n_{2} E_{0}}{Z_{0}},
\end{aligned}
$$

we obtain 


$$
\begin{aligned}
\vec{E}(\vec{r})= & \frac{i p_{2} E_{0}}{4 \pi R_{1}^{2}} \int \mathrm{d} a^{\prime} T_{p}\left[G_{1} f_{2}(\hat{r}\right. \\
& \left.\left.\times \vec{h}_{1}\right)-G_{2} f_{2}\left(\hat{r} \times \vec{e}_{1}\right) \times \vec{r}_{11}-G_{2} f_{2}\left(\hat{r} \cdot \vec{e}_{1}\right) \vec{r}_{11}\right], \\
\vec{H}(\vec{r})= & \frac{i p_{2} n_{2} E_{0}}{4 \pi Z_{0} R_{1}^{2}} \int \mathrm{d} a^{\prime} T_{p}\left[G_{1} f_{2}(\hat{r}\right. \\
& \left.\left.\times \vec{e}_{1}\right)+G_{2} f_{2}\left(\hat{r} \times \vec{h}_{1}\right) \times \vec{r}_{11}+G_{2} f_{2}\left(\hat{r} \cdot \vec{h}_{1}\right) \vec{r}_{11}\right] .
\end{aligned}
$$

Since the surface element is $\mathrm{d} a^{\prime}=R_{1}^{2} \sin \theta^{\prime} \mathrm{d} \theta^{\prime} \mathrm{d} \phi^{\prime}$, the expressions in Eqs. (33) and (34) can be simplified to

$$
E(\vec{r}) \equiv A \vec{U}(\vec{r})
$$

where

$$
\begin{gathered}
\vec{U}(\vec{r})=\int_{0}^{2 \pi} \int_{\pi}^{\pi-\theta_{m}} \sin \theta^{\prime} \mathrm{d} \theta^{\prime} \mathrm{d} \phi^{\prime}\left(\vec{u}_{1}+\vec{u}_{2}+\vec{u}_{3}\right), \\
H(\vec{r}) \equiv \frac{A n_{2}}{Z_{0}} \vec{V}(\vec{r})
\end{gathered}
$$

where

$$
\vec{V}(\vec{r})=\int_{0}^{2 \pi} \int_{\pi}^{\pi-\theta_{m}} \sin \theta^{\prime} \mathrm{d} \theta^{\prime} \mathrm{d} \phi^{\prime}\left(\vec{v}_{1}+\vec{v}_{2}+\vec{v}_{3}\right),
$$

with

$$
A=-\frac{i p_{2}}{4 \pi} E_{0}
$$

$\theta_{m}$ is the half-angle aperture, and the vectors $\vec{u}_{1}, \vec{u}_{2}, \vec{u}_{3}$, $\vec{v}_{1}, \vec{v}_{2}$, and $\vec{v}_{3}$ represent the three vectors of the three individual terms of $\vec{E}$ and $\vec{H}$ in Eqs. (10) and (11), respectively. Each of these vectors has the following components:

$$
\begin{gathered}
u_{1 x}=T_{p} f_{2} G_{1}\left(\cos \theta^{\prime} \cos ^{2} \phi^{\prime}+t_{1} \cos \theta_{t}^{\prime} \sin ^{2} \phi^{\prime}\right), \\
u_{1 y}=T_{p} f_{2} G_{1} \sin \phi^{\prime} \cos \phi^{\prime}\left(\cos \theta^{\prime}-t_{1} \cos \theta_{t}^{\prime}\right), \\
u_{1 z}=-T_{p} f_{2} G_{1} \sin \theta^{\prime} \cos \phi^{\prime}, \\
u_{2 x}=T_{p} f_{2} G_{2}\left[\left(z_{1}-z_{1}^{\prime}\right) \alpha_{1 y}-\left(y_{1}-y_{1}^{\prime}\right) \alpha_{1 z}\right], \\
u_{2 y}=T_{p} f_{2} G_{2}\left[\left(x_{1}-x_{1}^{\prime}\right) \alpha_{1 z}-\left(z_{1}-z_{1}^{\prime}\right) \alpha_{1 x}\right], \\
u_{2 z}=-T_{p} f_{2} G_{2}\left[\left(y_{1}-y_{1}^{\prime}\right) \alpha_{1 x}+\left(x_{1}-x_{1}^{\prime}\right) \alpha_{1 y}\right], \\
u_{3 x}=T_{p} f_{2} G_{2} \sin \theta_{t}^{\prime} \cos \phi^{\prime}\left(x_{1}-x_{1}^{\prime}\right), \\
u_{3 y}=T_{p} f_{2} G_{2} \sin \theta_{t}^{\prime} \cos \phi^{\prime}\left(y_{1}-y_{1}^{\prime}\right), \\
u_{3 z}=T_{p} f_{2} G_{2} \sin \theta_{t}^{\prime} \cos \phi^{\prime}\left(z_{1}-z_{1}^{\prime}\right),
\end{gathered}
$$

$$
\begin{gathered}
v_{1 x}=T_{p} f_{2} G_{1} \sin \phi^{\prime} \cos \phi^{\prime}\left(t_{1} \cos \theta^{\prime}-\cos \theta_{t}^{\prime}\right), \\
v_{1 y}=T_{p} f_{2} G_{1}\left(t_{1} \cos \theta^{\prime} \sin ^{2} \phi^{\prime}+\cos \theta_{t}^{\prime} \cos ^{2} \phi^{\prime}\right), \\
v_{1 z}=T_{p} f_{2} G_{1}\left(t_{1} \sin \theta^{\prime} \sin \phi^{\prime}\right), \\
v_{2 x}=T_{p} f_{2} G_{2}\left[\left(z_{1}-z_{1}^{\prime}\right) \beta_{1 y}-\left(y_{1}-y_{1}^{\prime}\right) \beta_{1 z}\right], \\
v_{2 y}=T_{p} f_{2} G_{2}\left[\left(x_{1}-x_{1}^{\prime}\right) \beta_{1 z}-\left(z_{1}-z_{1}^{\prime}\right) \beta_{1 x}\right], \\
v_{2 z}=T_{p} f_{2} G_{2}\left[\left(y_{1}-y_{1}^{\prime}\right) \beta_{1 x}-\left(x_{1}-x_{1}^{\prime}\right) \beta_{1 y}\right], \\
v_{3 x}=T_{p} f_{2} G_{2} t_{1} \sin \theta_{t}^{\prime} \sin \phi^{\prime}\left(x_{1}-x_{1}^{\prime}\right), \\
v_{3 y}=T_{p} f_{2} G_{2} t_{1} \sin \theta_{t}^{\prime} \sin \phi^{\prime}\left(y_{1}-y_{1}^{\prime}\right), \\
v_{3 z}=T_{p} f_{2} G_{2} t_{1} \sin \theta_{t}^{\prime} \sin \phi^{\prime}\left(z_{1}-z_{1}^{\prime}\right) .
\end{gathered}
$$

$\vec{\alpha}_{1}$ and $\vec{\beta}_{1}$, respectively, denote the $\hat{r} \times \vec{e}_{1}$ and $\hat{r} \times \vec{h}_{1}$ terms occurring in Eqs. (33) and (34). The components of $\vec{\alpha}_{1}$ and $\vec{\beta}_{1}$ are given by

$$
\begin{aligned}
& \alpha_{1 x}=\sin \phi^{\prime} \cos \phi^{\prime}\left(t_{1} \cos \theta^{\prime}-\cos \theta_{t}^{\prime}\right), \\
& \alpha_{1 y}=\cos \theta_{t}^{\prime} \cos ^{2} \phi^{\prime}+t_{1} \cos \theta^{\prime} \sin ^{2} \phi^{\prime}, \\
& \alpha_{1 z}=-t_{1} \sin \theta^{\prime} \sin \phi^{\prime}, \\
& \beta_{1 x}=-\cos \theta^{\prime} \cos ^{2} \phi^{\prime}-t_{1} \cos \theta_{t}^{\prime} \sin ^{2} \phi^{\prime}, \\
& \beta_{1 y}=\sin \phi^{\prime} \cos \phi^{\prime}\left(t_{1} \cos \theta_{t}^{\prime}-\cos \theta^{\prime}\right), \\
& \beta_{1 z}=\sin \theta^{\prime} \cos \phi^{\prime} .
\end{aligned}
$$

The coordinates $x_{1}, y_{1}, z_{1}, x_{1}^{\prime}, y_{1}^{\prime}$, and $z_{1}^{\prime}$ can be expressed in terms of the spherical coordinates as

$$
\begin{aligned}
& x_{1}=r_{1} \sin \theta \cos \phi, \\
& y_{1}=r_{1} \sin \theta \sin \phi, \\
& z_{1}=r_{1} \cos \theta, \\
& x_{1}^{\prime}=\sin \theta^{\prime} \cos \phi^{\prime}, \\
& y_{1}^{\prime}=\sin \theta^{\prime} \sin \phi^{\prime}, \\
& z_{1}^{\prime}=\cos \theta^{\prime},
\end{aligned}
$$

where $r_{1} \equiv r / R_{1}$.

The Poynting vector of the wave in the second medium can be written as

$$
\vec{S}_{2}=\operatorname{Re}\left(\vec{E} \times \vec{H}^{*}\right)=\frac{n_{2}}{Z_{0}}\left|E_{0}\right|^{2} \operatorname{Re}\left(\vec{U} \times \vec{V}^{*}\right)
$$

while the Poynting vector associated with the plane wave traveling in the first medium is given by 


$$
\vec{S}_{1}=\frac{n_{1}}{Z_{0}}\left|E_{0}\right|^{2} \hat{z}=S_{1} \hat{z}
$$

\section{CALCULATION OF ELECTROMAGNETIC FIELDS AND POYNTING VECTORS}

In Figs. 2 and 3, the maximum values of the $z$ component of $\vec{S}_{2}$, normalized to $S_{1}$, are plotted against the halfangular aperture, i.e., $\theta_{m}$. The maximization of $S_{2 z}$ is done with respect to the $x_{1}, y_{1}$, and $z_{1}$ coordinates for each value of $\theta_{m} . S_{2 z}$ maximizes on axis, i.e., at $x_{1}=y_{1}=0$, but not necessarily at the same values of $z_{1}$ for different $\theta_{m}$. The dashed curves in Figs. 2 and 3 show the values of the on-axis maximum irradiance obtained using the paraxial approximation, which is given by

$$
I_{p}=\left(\frac{2}{1+n}\right)^{2}\left(\frac{p_{2} \sin \theta_{m}}{2 f_{1}}\right)^{2},
$$

where

$$
f_{1}=\frac{n}{n-1}
$$

and the on-axis maximum is obtained at $z_{1}=f_{1}$. Equation (52) can be derived from the treatment in Ref. 1 .

In Fig. 2, the value of $p_{2}$ chosen is $10^{6}$ for two choices of $n$. In Fig. 3 the value of $n$ is chosen to be 1.5 for two choices of $p_{2}$. It is seen that the on-axis irradiance maximizes at a particular value of $\theta_{m}$, beyond which the effects of aberration become pronounced and the irradiance is limited to a value lower than that at the maximum. Thus,

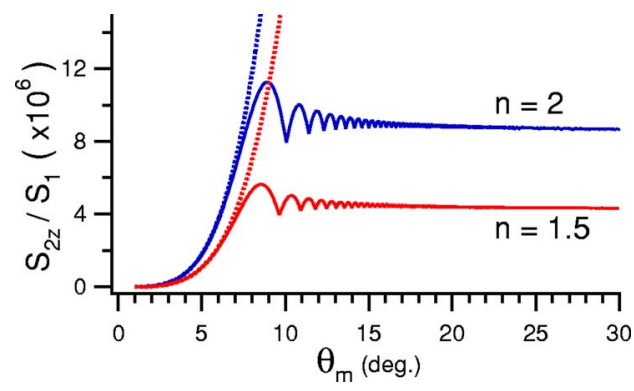

Fig. 2. (Color online) Maximum values of the normalized onaxis irradiance versus the half-angular aperture, $\theta_{m}$, for $p_{2}$ equal to $1 \times 10^{6}$. The upper and lower plots are for $n=1.5$ and 2 , respectively. The dashed curves are calculated using the paraxial approximation, Eq. (52).

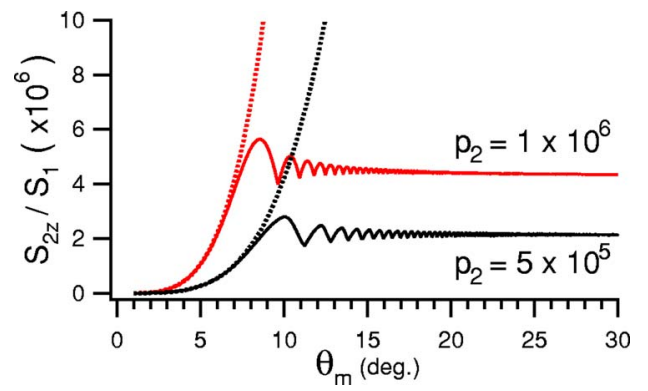

Fig. 3. (Color online) Maximum values of the normalized onaxis irradiance for $n=1.5$. The upper plots are for a $p_{2}$ value of $1 \times 10^{6}$, and the lower plots are for a $p_{2}$ value of $5 \times 10^{5}$. The dashed curves are the paraxial values calculated using Eq. (52).
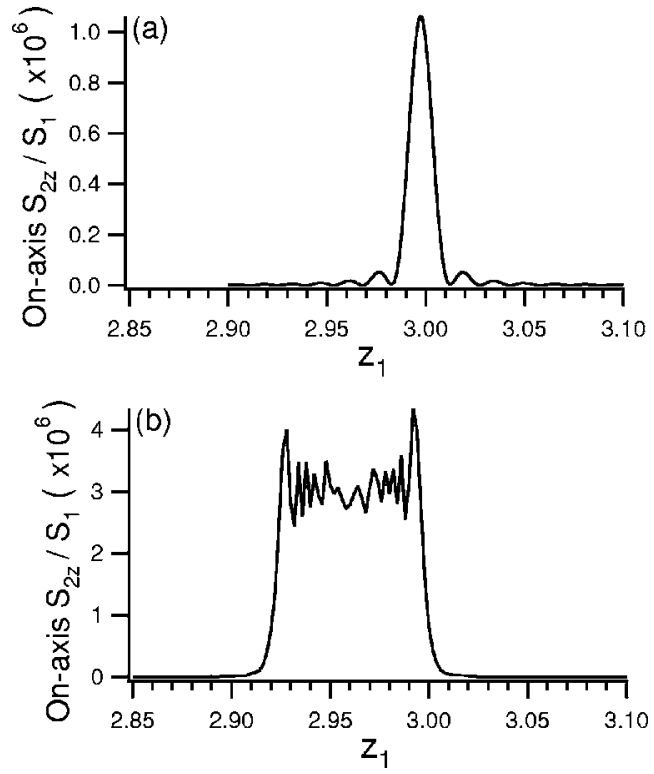

Fig. 4. Calculated on-axis irradiance versus axial position for (a) $\theta_{m}=5^{\circ}$ and (b) $\theta_{m}=20^{\circ}$, with $p_{2}$ value of $1 \times 10^{6}$ and $n=1.5$.
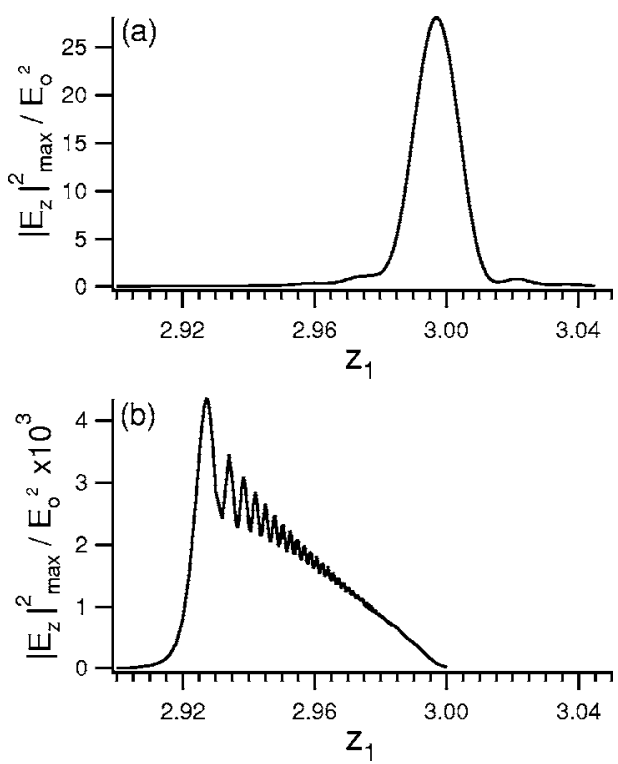

Fig. 5. Maximum calculated value of the modulus square of the longitudinal component of the electric field $\left(E_{z}\right)$ as a function of axial position for (a) $\theta_{m}=5^{\circ}$ and (b) $\theta_{m}=20^{\circ}$, with $n=1.5$ and $p_{2}$ $=10^{6}$. The value of $\left|E_{z}\right|^{2} / E_{0}^{2}$ displayed for each axial position is maximized with respect to $x_{1}$ and $y_{1}$.

when a spherical surface is used for focusing light, for given values of the refractive indices, wavelength of light, and radius of curvature of the surface, there exists an optimum aperture angle at which the highest irradiance can be obtained by focusing. For $p_{2}=10^{6}$ and $n=1.5$, the optimum value of $\theta_{m}$ is about $8.6^{\circ}$, which corresponds to an $f$ number of about 10. Thus, in this case, the paraxial approximation is valid only for $f$ numbers equal to 10 or larger. If the radius of curvature is reduced by a factor of 2 , i.e., for $p_{2}=5 \times 10^{5}$ and $n=1.5$, the optimum value of $\theta_{m}$ is about $10^{\circ}$, which corresponds to an $f$ number of about 8.6. In Fig. 4, the dependence of the (normalized) on-axis 
irradiance is plotted against the distance from the vertex for two values of $\theta_{m}$. At $\theta_{m}=5^{\circ}$, the wave focuses at a normalized distance of 3 , in accordance with the paraxial result in Eq. (53) as well as that obtained from the ray tracing. ${ }^{9}$ At $\theta_{m}=20^{\circ}$, however, the on-axis irradiance is not as sharply focused, and the region of high irradiance extends over a larger axial region $\left(z_{1}=2.94\right.$ to 3$)$.

In Fig. 5, the maximum values of the normalized energy density associated with the longitudinal field component $E_{z}$ are plotted as a function of $z_{1}$. For each $z_{1}$ shown in Fig. 5, the value of $\left|E_{z}\right|^{2}$ is maximized with respect to $x_{1}$ and $y_{1}$ variables. For $\theta_{m}=5^{\circ}$, it is found that $\left|E_{z}\right|^{2}$ maximizes at the axial position of the geometrical focus (i.e., at $\left.z_{1} \approx 3\right)$. As the beam comes into focus, the longitudinal component of the Poynting vector increases. The value of the longitudinal component maximizes slightly off axis and is zero for all axial points by symmetry. For $\theta_{m}=20^{\circ}$, spherical aberration has become significant, and $\left|E_{z}\right|^{2}$ maximizes over a much larger axial range, reaching a global maximum at a closer location of $z_{1} \simeq 2.93$. Figure 5 also shows that, at $\theta_{m}=20^{\circ}$, the normalized value of $\left|E_{z}\right|^{2} / E_{0}^{2}$ reaches a maximum value of $\sim 4350$, whereas the
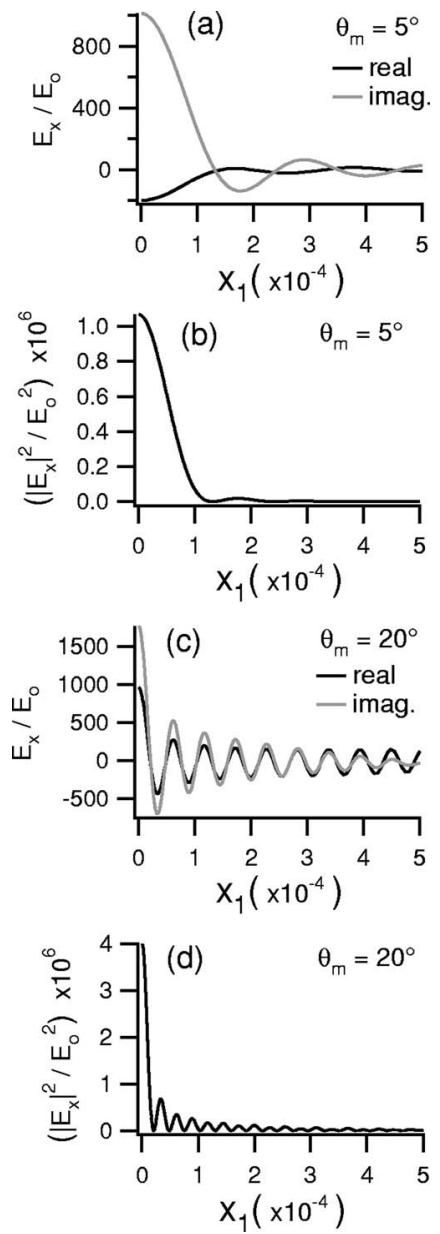

Fig. 6. Plots of the real (black) and imaginary (gray) parts and the modulus squared (black) of the $x$ component of the electric field versus $x_{1}$ for $\theta_{m}$ equal to (a) and (b) $5^{\circ}$ and (c) and (d) $20^{\circ}$, respectively. All plots are for $p_{2}=10^{6}, n=1.5$, and $y_{1}=0 . z_{1}$ for each plot is chosen such that the on-axis value of $\left|E_{x}\right|^{2}$ is maximum at that $z_{1}$; i.e., for $\theta_{m}=5^{\circ}, z_{1}=2.997$ and, for $\theta_{m}=20^{\circ}, z_{1}$ $=2.927$.
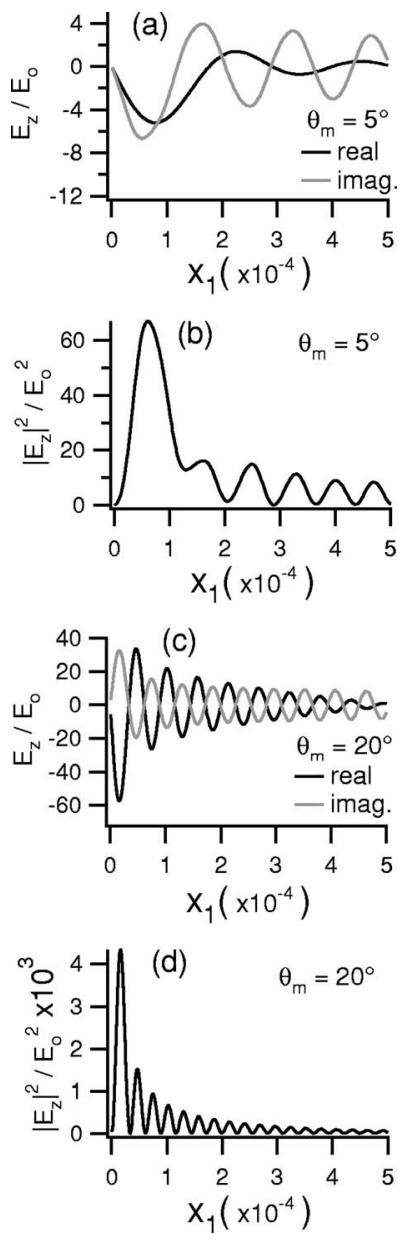

Fig. 7. Plots of the real part (black) and imaginary part (gray) and modulus squared (black) of the normalized longitudinal component, $E_{z} / E_{0}$, for (a) and (b) $\theta_{m}=5^{\circ}$ and (c) and (d) $20^{\circ}$, respectively. For each plot $p_{2}=10^{6}, n=1.5$, and $y_{1}=0$. In each graph, $z_{1}$ is chosen such that $\left|E_{z}\right|^{2}$ is maximum; i.e., for $\theta_{m}=5^{\circ}, z_{1}=2.997$, and, for $\theta_{m}=20^{\circ}, z_{1}=2.927$.

maximum value of $S_{z} / S_{1}$ is about $4 \times 10^{6}$ for $p_{2}=10^{6}, n$ $=1.5$, and $z_{1}=2.927$, i.e., the longitudinal irradiance is about $0.1 \%$ of the transverse irradiance.

The radial extents of the transverse and the longitudinal fields (and irradiances) are shown in Figs. 6 and 7. These figures show that both $\left|E_{x}\right|^{2}$ and $\left|E_{z}\right|^{2}$ are mostly confined to within $x_{1} \approx 10^{-4}$, although $\left|E_{x}\right|^{2}$ maximizes on axis, whereas $\left|E_{z}\right|^{2}$ is zero for on-axis points and has its maximum value on the $x$ axis, i.e., $x_{1} \approx 5 \times 10^{-5}$ and $y_{1}$ $=0$.

To determine the relative contributions of the three terms in the integrands of Eqs. (36) and (38), which correspond to the three terms in the integrands of Eqs. (10) and (11), we show in Tables 1-4 the squares of the absolute values of all the Cartesian components of $u_{1}, u_{2}, u_{3}$, and $v_{1}, v_{2}, v_{3}$ maximized with respect to $x_{1}, y_{1}$, and $z_{1}$, for $p_{2}=10^{6}, n=1.5$, and $\theta_{m}=5^{\circ}$ and $20^{\circ}$. From the tables we find that the dominant contributions to the fields near the focus come from the field components parallel to the incident polarization directions (i.e., $E_{x}$ and $H_{y}$ ). However, the components perpendicular to the incident fields (i.e., $E_{y}$, $E_{z}, H_{x}$, and $H_{z}$ ) also achieve nonnegligible values in the region near the focus. We also find that, for $E_{x}$ and $H_{y}$, 
both of the first two terms (i.e., $u_{1}$ and $u_{2}, v_{1}$ and $v_{2}$ ) are important, whereas the contribution of the third term is small. However, for the longitudinal components, the third terms $\left(u_{3}\right.$ and $\left.v_{3}\right)$ contribute dominantly.

The values of the modulus squares of the individual components of the terms given in Tables 1 and 2 are much different from the values of the modulus square of the fields in Figs. 2-7 because the individual components interfere constructively or destructively. Tables 1 and 2 also illustrate the increases of each of the components of the three vector terms for $\vec{E}$ and $\vec{H}$ for larger collection angles. For the electric field, the increase of the $x$ component is disproportionately larger for the third integral term, whereas the increase in the $y$ component is disproportionately larger for the first and third integral terms. For the magnetic field, the increase of the $y$ component is disproportionately larger for the third integrand term, and the increase in the $x$ component is disproportionately larger for the first and third integrand terms. The increase in the longitudinal component of both the electric and the magnetic fields is fairly even from all three integral terms. Thus, the effects of the increasing spherical

Table 1. Global Maxima of the $x, y$, and $z$ Components of the Three Vector Terms of the Calculated Electric Fields for $p_{2}=10^{6}, n=1.5$, and $\theta_{m}=5^{\circ}$ and $20^{\circ} a$

\begin{tabular}{cccc}
\hline Component & $\begin{array}{c}\text { Global Maximum } \\
\theta_{m}=5^{\circ}\end{array}$ & $\begin{array}{c}\text { Global Maximum } \\
\theta_{m}=20^{\circ}\end{array}$ & Ratio \\
\hline$\left|u_{1 x}\right|^{2}$ & $1.14 \times 10^{5}$ & $3.76 \times 10^{5}$ & $3.31 \times 10^{0}$ \\
$\left|u_{2 x}\right|^{2}$ & $3.24 \times 10^{5}$ & $1.09 \times 10^{6}$ & $3.37 \times 10^{0}$ \\
$\left|u_{3 x}\right|^{2}$ & $7.19 \times 10^{-2}$ & $2.62 \times 10^{2}$ & $3.64 \times 10^{3}$ \\
$\left|u_{1 y}\right|^{2}$ & $4.01 \times 10^{-3}$ & $1.71 \times 10^{1}$ & $4.27 \times 10^{3}$ \\
$\left|u_{2 y}\right|^{2}$ & $5.89 \times 10^{2}$ & $4.12 \times 10^{3}$ & $7.00 \times 10^{0}$ \\
$\left|u_{3 y}\right|^{2}$ & $1.41 \times 10^{-2}$ & $6.19 \times 10^{1}$ & $4.38 \times 10^{3}$ \\
$\left|u_{1 z}\right|^{2}$ & $1.12 \times 10^{2}$ & $1.70 \times 10^{4}$ & $1.51 \times 10^{2}$ \\
$\left|u_{2 z}\right|^{2}$ & $4.40 \times 10^{1}$ & $6.99 \times 10^{3}$ & $1.59 \times 10^{2}$ \\
$\left|u_{3 z}\right|^{2}$ & $1.76 \times 10^{2}$ & $2.69 \times 10^{4}$ & $1.53 \times 10^{2}$ \\
\hline
\end{tabular}

${ }^{a}$ Ratio denotes the ratio of the global maximum of that component for $\theta=20^{\circ}$ to the global maximum value for $\theta_{m}=5^{\circ}$.

Table 2. Global Maxima of the $x, y$, and $z$ Components of the Three Vector Terms of the Calculated Magnetic Fields for $p_{2}=10^{6}, n=1.5$, and $\theta_{m}=5^{\circ}$ and $20^{\circ} a$

\begin{tabular}{cccc}
\hline Component & $\begin{array}{c}\text { Global Maximum } \\
\theta_{m}=5^{\circ}\end{array}$ & $\begin{array}{c}\text { Global Maximum } \\
\theta_{m}=20^{\circ}\end{array}$ & Ratio \\
\hline$\left|v_{1 x}\right|^{2}$ & $4.57 \times 10^{-2}$ & $1.95 \times 10^{2}$ & $4.27 \times 10^{3}$ \\
$\left|v_{2 x}\right|^{2}$ & $9.36 \times 10^{3}$ & $5.59 \times 10^{4}$ & $5.97 \times 10^{0}$ \\
$\left|v_{3 x}\right|^{2}$ & $1.41 \times 10^{-2}$ & $6.19 \times 10^{1}$ & $4.38 \times 10^{3}$ \\
$\left|v_{1 y}\right|^{2}$ & $5.73 \times 10^{5}$ & $2.22 \times 10^{6}$ & $3.88 \times 10^{0}$ \\
$\left|v_{2 y}\right|^{2}$ & $3.23 \times 10^{5}$ & $1.31 \times 10^{6}$ & $4.05 \times 10^{0}$ \\
$\left|v_{3 y}\right|^{2}$ & $7.19 \times 10^{-2}$ & $2.62 \times 10^{2}$ & $3.64 \times 10^{3}$ \\
$\left|v_{1 z}\right|^{2}$ & $5.69 \times 10^{2}$ & $8.50 \times 10^{4}$ & $1.49 \times 10^{2}$ \\
$\left|v_{2 z}\right|^{2}$ & $2.21 \times 10^{1}$ & $3.55 \times 10^{3}$ & $1.61 \times 10^{2}$ \\
$\left|v_{3 z}\right|^{2}$ & $1.76 \times 10^{2}$ & $2.68 \times 10^{4}$ & $1.53 \times 10^{2}$ \\
\hline
\end{tabular}

${ }^{a}$ Ratio denotes the ratio of the global maximum of that component for $\theta=20^{\circ}$ to the global maximum value for $\theta_{m}=5^{\circ}$.
Table 3. Normalized Locations $\left(x_{1}, y_{1}, z_{1}\right)$ of the Global Maxima of the Three Vector Terms of the

Calculated Electric Fields for $p_{2}=10^{6}, n=1.5$, and $\theta_{m}=5^{\circ}$ and $20^{\circ}$

\begin{tabular}{|c|c|c|}
\hline Component & $\begin{array}{c}\text { Global Maximum } \\
\text { Location } \\
\left(x_{1}, y_{1}, z_{1}\right) \\
\theta_{m}=5^{\circ}\end{array}$ & $\begin{array}{c}\text { Global Maximum } \\
\text { Location } \\
\left(x_{1}, y_{1}, z_{1}\right) \\
\theta_{m}=20^{\circ}\end{array}$ \\
\hline$\left|u_{1 x}\right|^{2}$ & $(0,0,2.997)$ & $(0,0,2.9717)$ \\
\hline$\left|u_{2 x}\right|^{2}$ & $(0,0,2.997)$ & $(0,0,2.9717)$ \\
\hline$\left|u_{3 x}\right|^{2}$ & $(0,0,2.997)$ & $(0,0,2.9272)$ \\
\hline$\left|u_{1 y}\right|^{2}$ & $\begin{array}{c}\left(8.8 \times 10^{-5}, 8.6 \times 10^{-5}\right. \\
2.997)\end{array}$ & $\begin{array}{c}\left(1.9 \times 10^{-5}, 1.9 \times 10^{-5}\right. \\
2.9273)\end{array}$ \\
\hline$\left|u_{2 y}\right|^{2}$ & $\begin{array}{c}\left(9.6 \times 10^{-5}, 9.5 \times 10^{-5}\right. \\
2.997)\end{array}$ & $\begin{array}{c}\left(1.9 \times 10^{-5}, 1.9 \times 10^{-5}\right. \\
2.9274)\end{array}$ \\
\hline$\left|u_{3 y}\right|^{2}$ & $\begin{array}{c}\left(8.7 \times 10^{-5}, 8.8 \times 10^{-5}\right. \\
2.997)\end{array}$ & $\begin{array}{c}\left(1.9 \times 10^{-5}, 1.9 \times 10^{-5}\right. \\
2.9273)\end{array}$ \\
\hline$\left|u_{1 z}\right|^{2}$ & $\left(7.9 \times 10^{-5}, 0,2.997\right)$ & $\left(1.62 \times 10^{-5}, 0,2.9273\right)$ \\
\hline$\left|u_{2 z}\right|^{2}$ & $\left(7.9 \times 10^{-5}, 0,2.997\right)$ & $\left(1.62 \times 10^{-5}, 0,2.9272\right)$ \\
\hline$\left|u_{3 z}\right|^{2}$ & $\left(7.9 \times 10^{-5}, 0,2.997\right)$ & $\left(1.61 \times 10^{-5}, 0,2.9272\right)$ \\
\hline
\end{tabular}

Table 4. Normalized Locations $\left(x_{1}, y_{1}, z_{1}\right)$ of the Global Maxima of the Three Vector Terms of the Calculated Magnetic Fields for $p_{2}=10^{6}, n=1.5$, and $\theta_{m}=5^{\circ}$ and $20^{\circ}$

\begin{tabular}{ccc}
\hline & $\begin{array}{c}\text { Global Maximum } \\
\text { Location } \\
(x, y, z)\end{array}$ & $\begin{array}{c}\text { Global Maximum } \\
\text { Location } \\
(x, y, z) \\
\theta_{m}=5^{\circ}\end{array}$ \\
\hline Component & $\left(8.8 \times 10^{-5}, 8.8 \times 10^{-5}\right.$, & $\left(1.9 \times 10^{-5}, 1.9 \times 10^{-5}\right.$, \\
& $2.997)$ & $2.9272)$ \\
$\left|v_{1 x}\right|^{2}$ & $\left(9.6 \times 10^{-5}, 9.6 \times 10^{-5}\right.$, & $\left(1.9 \times 10^{-5}, 1.9 \times 10^{-5}\right.$, \\
$\left|v_{2 x}\right|^{2}$ & $2.997)$ & $2.9272)$ \\
$\left|v_{3 x}\right|^{2}$ & $\left(8.7 \times 10^{-5}, 8.8 \times 10^{-5}\right.$, & $\left(1.9 \times 10^{-5}, 1.9 \times 10^{-5}\right.$, \\
$\left|v_{1 y}\right|^{2}$ & $2.997)$ & $2.9272)$ \\
$\left|v_{2 y}\right|^{2}$ & $(0,0,2.997)$ & $(0,0,2.9272)$ \\
$\left|v_{3 y}\right|^{2}$ & $(0,0,2.997)$ & $(0,0,2.9272)$ \\
$\left|v_{1 z}\right|^{2}$ & $(0,0,2.997)$ & $(0,0,2.9272)$ \\
$\left|v_{2 z}\right|^{2}$ & $\left(0,7.5 \times 10^{-5}, 2.997\right)$ & $\left(0,1.62 \times 10^{-5}, 2.9275\right)$ \\
$\left|v_{3 z}\right|^{2}$ & $\left(0,8.0 \times 10^{-5}, 2.997\right)$ & $\left(0,1.52 \times 10^{-5}, 2.9275\right)$ \\
\hline
\end{tabular}

aberration on the beam distributions in the focal region cannot be attributed to a single term in Eq. (10) or (11) but rather manifest themselves from all three terms.

Tables 3 and 4 illustrate the locations of the global maxima of the Cartesian components of all three of the integral terms for $\vec{E}$ and $\vec{H}$ given in Tables 1 and 2 .

\section{CONCLUSIONS}

We have derived and evaluated the integrals describing the electromagnetic fields when a plane wave of light is refracted by a spherical surface. The integrals are expressed in terms of dimensionless variables and dimensionless physical parameters, so that the results are applicable over a wide range of wavelengths. The result allows us to calculate the maximum values of irradiances achievable in the presence of spherical aberrations and 
also show the distribution and the relative contributions of the longitudinal component of the fields.

S. Guha, the corresponding author, can be reached by e-mail at shekhar.guha@wpafb.af.mil.

*Current address, Physics Department, California Polytechnic State University, San Luis Obispo, California 93407

\section{REFERENCES}

1. J. W. Goodman, Fourier Optics (Roberts, 2005), Chap. 5.

2. B. Richards and W. Wolf, "Electromagnetic diffraction in optical systems II," Proc. R. Soc. London Ser. A 253, 353-379 (1959).
3. T. D. Visser and S. H. Wiersma, "Diffraction of converging electromagnetic waves," J. Opt. Soc. Am. A 9, 2034-2047 (1992).

4. W. Hsu and R. Barakat, "Stratton-Chu vectorial diffraction of electromagnetic fields by apertures with application to small-Fresnel-number systems," J. Opt. Soc. Am. A 11, 623-629 (1994).

5. J. A. Stratton and L. J. Chu, "Diffraction theory of electromagnetic waves," Phys. Rev. 56, 99-107 (1939).

6. J. A. Stratton, Electromagnetic Theory (McGraw-Hill, 1941).

7. M. Born and E. Wolf, Principles of Optics (Cambridge U. Press, 2003).

8. E. J. Rothwell and E. J. Cloud, Electromagnetics (CRC Press, 2001).

9. F. A. Jenkins and H. E. White, Fundamentals of Optics (McGraw-Hill, 1957). 